\title{
In our experience: An audit of halitosis symptoms in patients with SIGN compliant recurrent tonsillitis
}

\author{
Catriona Douglas ${ }^{1}$, Ruth Begbie ${ }^{2}$, David Young ${ }^{3}$, and Kenneth MacKenzie ${ }^{1}$ \\ ${ }^{1}$ Queen Elizabeth University Hospital \\ ${ }^{2}$ University of Glasgow \\ ${ }^{3}$ Strathclyde University
}

October 6, 2020

Key Points

- The mean TOI-14 was 48.22 (SD 10.10).

- The mean HALT was 45.80 (SD 22.22).

- There was a significant correlation between the TOI-14 and HALT, $r=0.605, \mathrm{p}<0.001$.

- This is the first evidence that patients with poor quality of life due to sore throat also have poor quality of life due to halitosis.

- It is currently unknown if the HALT score is modified by tonsillectomy.

\section{Background}

Recurrent tonsillitis remains one of the most common problems that GPs manage, with an annual reported incidence of 100 per 1000 population ${ }^{1}$. Recurrent tonsillitis can have a significant impact on a patient's quality of life; repeated absences from nursery/school/work and hospital admissions can cause a serious negative impact on both the patient and their family ${ }^{2}$.

Halitosis is a general term used to define an unpleasant or offensive odour emanating from expired air, which can originate from both oral and non-oral sources. Halitosis is a relatively common disorder, with $10-30 \%$ of the population said to report it, with the prevalence being equal between females and males ${ }^{3}$. Halitosis has multifactorial origins, most commonly related to pathology in the oral cavity, oropharynx or respiratory disease. It is mostly due to putrefying bacteria present on the dorsum of the tongue and the volatile sulphur compounds (VSC's) produced from food elements. Halitosis has significant social and psychological impacts.

Despite very many reports suggesting that tonsillitis causes halitosis, there is very little published literature to support this. The aim of this audit was to establish the prevalence of halitosis symptoms in patients who are SIGN compliant for tonsillectomy due to recurrent tonsillitis.

\section{Materials and Methods}

Patient Questionnaires

A prospective observational cohort audit of patients who fulfilled the Scottish Intercollegiate Guidelines Network (SIGN) criteria for tonsillectomy was performed. Patients were recruited when they attended for assessment of tonsillectomy. All patients were aged 16 or above. All patients were invited to complete two questionnaires after they had been added to the waiting list for tonsillectomy. The Tonsil Outcome Inventory-14 (TOI14) is a validated disease specific tool used to assess the quality of life impact of tonsillitis ${ }^{5}$. It distinguishes normal healthy subjects from chronic tonsillitis sufferers with high sensitivity. 
The Halitosis Associated Life-quality Test (HALT) is a specific 20-item quality of life measure for halitosis. Is describes the burden of halitosis and is sensitive to change ${ }^{6}$. HALT scores are significantly associated with the scales of organoleptic testing, regarded as the 'gold standard' measurement for halitosis ${ }^{7}$.

Smoking status was recorded from the ENT history. Patients were regarded as having reflux if they were taking anti reflux medication.

\section{Statistical analysis}

The relationship between numerical variables and HALT and TOI14 was assessed using correlations. Differences between HALT and TOI14 between levels of categorical variables were tested using t-tests for two groups and ANOVA more than two groups. Where the data were not normally distributed, non-parametric tests were used. All analyses were done using Minitab (version 19) with a $5 \%$ significance level.

\section{Results}

A total of 65 patients were recruited to the audit between Aug 2019 - Oct 2019, at the Glasgow Royal Infirmary ENT department. Six patients were excluded due to incomplete questionnaires, leaving 59 patients for analysis. All patients fulfilled the SIGN criteria for tonsillectomy due to recurrent tonsillitis. There were 45 females and 15 males. The mean age was 23 years (range 16-44). see table 1.

\section{Questionnaires}

The mean TOI-14 was 48.22 (SD 10.10), the mean HALT was 45.80 (SD 22.22). There was a significant correlation between the TOI-14 and HALT, $r=0.605, \mathrm{p}<0.001$, see figure 1 .

There was no evidence of a correlation between TOI-14 and age or BMI. There was no evidence of a difference in the mean TOI-14 between those with or without reflux. There was no evidence of difference in the mean TOI-14 between the three smoking groups. There was some evidence that the TOI14 was higher in females but this did not reach statistical significance at the $5 \%$ level $-\mathrm{p}=0.064$. There was no correlation between HALT and age or BMI. There was no difference in the mean HALT between males and females. There was no evidence of a difference in the mean HALT between the three smoking categories. There was no evidence of a difference in the mean HALT between those with and without reflux.

\section{Discussion}

This study has demonstrated a significant correlation between recurrent tonsillitis and halitosis. Tonsillitis is a very common clinical condition, with tonsillectomy being performed when patients suffer from repeated disabling episodes ${ }^{4}$. Recurrent tonsillitis causes significant morbidity for the patient including time of work, with a major impact on a patient's quality of life ${ }^{8}$. The TIO-14 is a disease specific quality of life tool for assessing sore throat. In this study the mean TOI-14 score was 48.22, comparable to Skevas et al and Roplekar et $\mathrm{al}^{5,9}$. Laajala et al demonstrated that a TOI-14 score of greater than 40 suggests a patient has severe symptoms of tonsillitis. There was no evidence of correlation between the TIO-14 and sex, BMI, reflux or smoking status comparable to the findings by Laajala et al.

Although oropharyngeal pathology is frequently listed as a cause for halitosis, there is little published literature to support this. The few papers that discuss halitosis in relation to oropharyngeal pathology tend to highlight tonsoliths as the cause of halitosis, rather than provide evidence that tonsillitis is the cause. In this paper only 3 patients had tonsil stones, and this was not significantly related to the HALT score. The HALT has never been used in patients with recurrent tonsillitis. Halitosis can significantly impact on a patient's quality of life, with it being considered one of the most socially unattractive aspects of social interactions ${ }^{10}$. Patients can have low self-esteem, anxiety, depression and poor self-confidence. The halitosis can also impact on relatives and friends as they may need to reassure or counsel the sufferer about their bad breath. There is also a large economic burden associated with halitosis, with Meninguad et al reporting that over 2 billion dollars is spent annually on products to mask halitosis ${ }^{11}$. Therefore a considerable amount of psychological, social and economic resources are dedicated to patients with halitosis, in a similar way to those with recurrent tonsillitis. The HALT was not associated with smoking, although smoking is 
commonly linked to severity of halitosis. Halitosis is not an indicator for tonsillectomy in the UK, however some countries list it as a relative indication. This is the first evidence that patients with poor quality of life due to sore throat also have poor quality of life due to halitosis.

The significant correlation of the TIO-14 and HALT could be attributed to the questionnaires asking the same questions, however there is only one question that is the same in both questionnaires - patients are asked about sore throat. There is no overlap between the other questions.

It is well established that tonsillectomy improves the TIO-14 score in patients. It is currently unknown if the HALT score is modified by tonsillectomy.

Data Availability Statement

Data available from authors on request.

\section{References}

1. Shvartzman P. Are antibiotics appropriate for sore throats? Careful prescribing is beneficial. BMJ. 1994;309(6960):1011-1012.

2. Torretta S, Rosazza C, Pace ME, Iofrida E, Marchisio P. Impact of adenotonsillectomy on pediatric quality of life: review of the literature. Ital J Pediatr. 2017;43(1):107.

3. Bollen CM, Beikler T. Halitosis: the multidisciplinary approach.Int J Oral Sci. 2012;4(2):55-63.

4. SIGN. Management of sore throat and indication of tonsillectomy. SIGN 117. . 2010; http://www.sign.ac.uk/guidelines/fulltext/117/ . .

5. Skevas T, Klingmann C, Plinkert PK, Baumann I. [Development and validation of the Tonsillectomy Outcome Inventory 14]. HNO.2012;60(9):801-806.

6. Kizhner V, Xu D, Krespi YP. A new tool measuring oral malodor quality of life. Eur Arch Otorhinolaryngol. 2011;268(8):1227-1232.

7. van den Broek AM, Feenstra L, de Baat C. A review of the current literature on aetiology and measurement methods of halitosis. J Dent. 2007;35(8):627-635.

8. Douglas CM, Lang K, Whitmer WM, Wilson JA, Mackenzie K. The effect of tonsillectomy on the morbidity from recurrent tonsillitis. Clin Otolaryngol. 2017.

9. Roplekar R, Van M, Hussain S. "Does the Quality of Life (QOL) impact of tonsillitis correlate with SIGN guideline compliance? An assessment of range and normality". Clin Otolaryngol. 2015.

10. de Jongh A, van Wijk AJ, Horstman M, de Baat C. Attitudes towards individuals with halitosis: an online cross sectional survey of the Dutch general population. Br Dent J. 2014;216(4):E8.

11. Meningaud JP, Bado F, Favre E, Bertrand JC, Guilbert F. [Halitosis in 1999]. Rev Stomatol Chir Maxillofac. 1999;100(5):240-244.

Table 1. Patient characteristics.

\begin{tabular}{lll}
\hline Characteristic & Characteristic & Number (range) \\
\hline Mean Age & Mean Age & $23(16-44)$ \\
Mean BMI & Mean BMI & $29(18-53)$ \\
& & Number $(\%)$ \\
Sex & Male & $14(24)$ \\
Smoking status & Female & $45(76)$ \\
& Yes & $14(24)$ \\
& No & $37(63)$ \\
& Ex & $7(13)$
\end{tabular}




\begin{tabular}{lll}
\hline Characteristic & Characteristic & Number (range) \\
\hline \multirow{3}{*}{ Reflux } & Missing & $1(2)$ \\
& Yes & $9(16)$ \\
\multirow{4}{*}{ Tonsil Stones } & No & $49(83)$ \\
& Missing & $1(1)$ \\
& Yes & $3(5)$ \\
& No & $56(95)$ \\
\hline
\end{tabular}

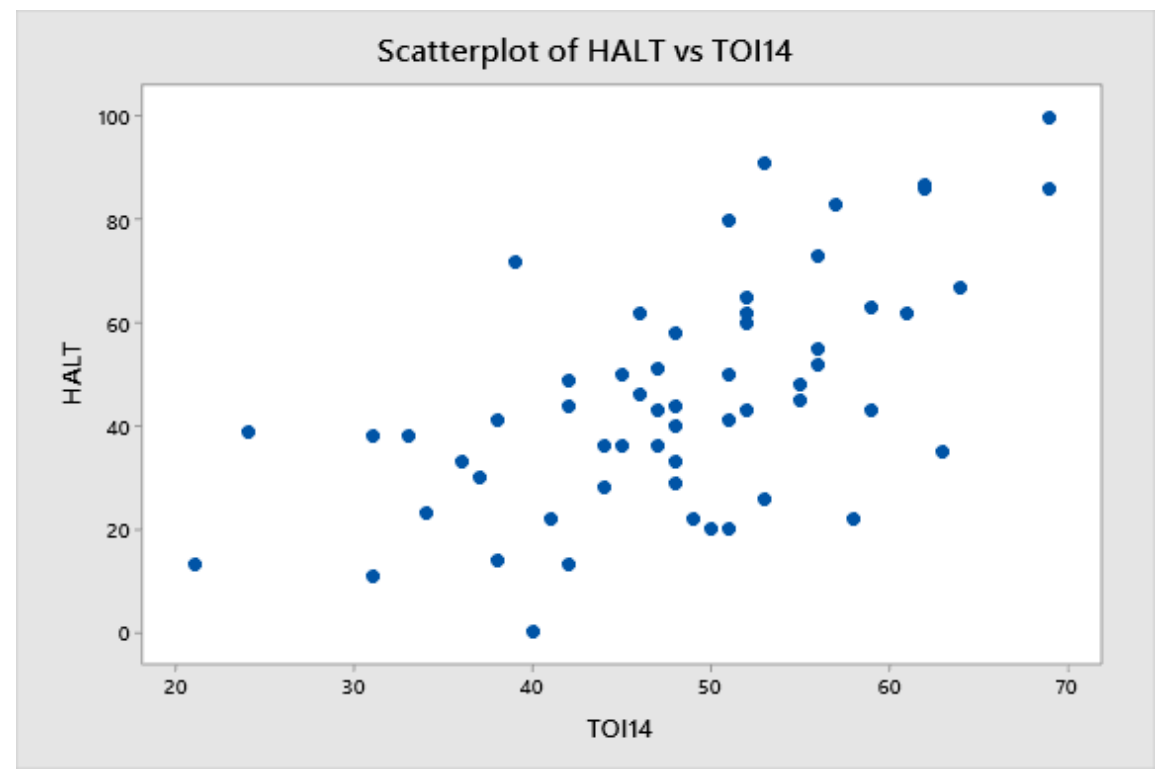

Figure 1. Scatterplot of HALT Vs TOI-14, demonstrating a significant correlation

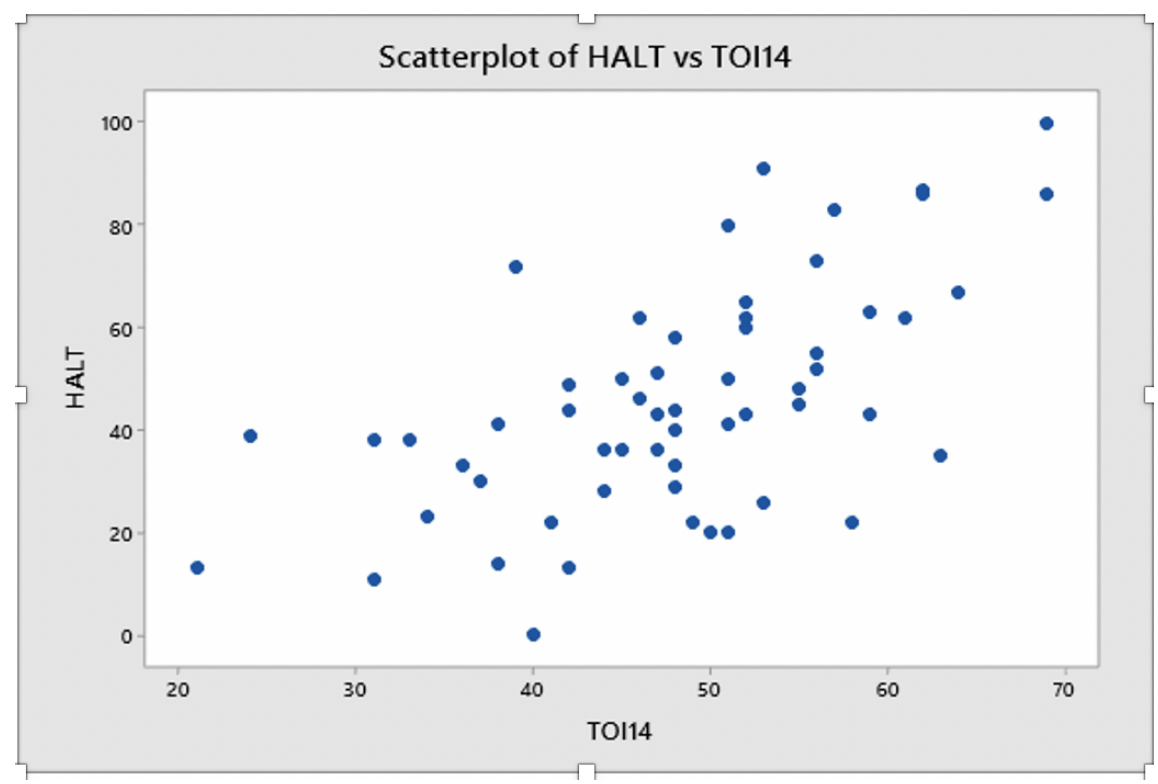

